\title{
Correspondence
}

\section{Specific risk factors of spinal epidural haematoma}

To the Editor:

The brief review by Wulf ${ }^{1}$ in the Journal is on a topic which all anaesthetists who use epidural techniques, view with grave concern. Spinal haematoma carries with it a rare but well-recognised risk of cord damage and paralysis or permanent anaesthesia. I therefore read the review with great interest. I was surprised to see that "epidural catheter inserted during general anaesthesia" was noted in the abstract findings as a risk factor. On reading the review it appears that what was meant was that in 3 of the 51 reports the epidural was inserted while the patient was under general anaesthesia. This cannot be a risk factor unless the relative frequency of the use of general anaesthesia with epidural is known. The same argument applies to the other "risk factors" such as the use of low molecular weight heparin (two cases), and indeed this "risk factor" seems to be discounted in the discussion - "it is unlikely that low molecular weight heparin present a special risk."

It is a great pity that Dr. Wulf, after going to the trouble of collecting as many cases as possible, then fails to analyse them critically and consistently. One further example will suffice. In Table II, six cases of haematoma in 1,334,506 patients are given (an incidence of 1 in 222,420 ), yet the text on page 1266 says seven cases and an incidence of 190,000 ).

I am very disappointed that such an important topic seems to have been given such an uncritical review which is more likely to mislead than assist practitioners.

W.J. Russell

Adelaide, South Australia

\section{REFERENCES}

I Wulf $H$. Epidural anaesthesia and spinal haematoma.

Can J Anaesth 1996; 43: 1260-71.

\section{REPLY}

$I$ appreciate very much the opportunity to respond to Dr. Russell's letter and clarify some points he raised. In Table II seven spinal haematomas are given, in accordance with the statement in the text.

As Dr Russell correctly states, it is not possible to give exact figures for risk ratios for any of the factors involved, since the relative frequency of the use of epidural anaesthesia in combination with potential "risk factors" is not known. As a consequence, the review gives the facts and lists the cases individually. This is not "misleading" but allows readers to draw their own conclusions. Nevertheless, some (qualitative) assumptions are offered.

Since the combination of ankylosing spondylitis or fibrinolytic therapy and epidural catheter insertion is uncommon, even a few reports of complications should raise attention. The same is true for application of epidurals during general anaesthesia, since this practice has been discouraged very intensively in many countries. On the other hand, at least in Europe, many anaesthetists use epidural catheters in patients after low dose heparin prophylaxis or in patients receiving NSAIDs. Millions of patients take aspirin during the week before surgery, often without remembering or reporting it to their anaesthetists. There are very few reports of complications associated with common factors such as NSAIDs or low dose heparin leading to the assumption given in the review that this is "probably indicating no increased risk."

Priv. Doz. Dr. med. Hinnerk Wulf Kiel, Germany

\section{Combined epidural/general anaesthesia and postoperative outcome}

To the Editor:

Dr. Carli's reasoned criticism ${ }^{1}$ of the paper by Garnett et al. on perioperative ischaemia in aortic surgery ${ }^{2}$ has not been satisfactorily addressed by the retort "Nothing could be further from the truth." 3 The hypothesis at issue is deceptively simple: that continued afferent neural blockade will prevent harmful reflex responses to major surgery. Contrary to Dr. Garnett's assertion, this hypothesis was not tested; effective bilateral epidural blockade was not verified from the time of incision onwards, and motor responses to possible inadequacies of segmental analgesia were masked by full curarizing doses of pancuronium. Postoperatively, steps were not taken to ensure that the segmental level of blockade remained above T5 bilaterally for the duration of the experimental period.

Dr. Garnett's article now joins a distinguished group of elegant, peer-reviewed papers in major journals, all suffering from the same basic flaw of reliance on muscle 
relaxants to mask inadequacies of regional anaesthesia, and all failing to test their hypothesis. ${ }^{4}$ The time is long overdue to review the design of experimental protocols in this very difficult area of nociception and clinical outcome, and to throw away the deceptive crutch of muscle relaxants, as some of us have done, with great satisfaction for major intrathoracic and abdominal surgery and ultra-early ambulation. ${ }^{5}$ Clearly, there is always a need for brief use of a short-acting muscle relaxant, such as succinylcholine, while diathermy-cautery is being used directly on the muscles of the abdominal or chest wall, but that is the only concession that should be made to reliance on the integrity of regional blockade for effective muscular relaxation, a quiet operative field and ultra-early mobilisation.

Philip R. Bromage MBBS FFARCS FRCPC

Montgomery Center, Vermont, U.S.A.

\section{REFERENCES}

1 Carli F, Klubien K, Baker C. Combined epidural/general anaesthesia (Letter). Can J Anaesth 1997; 44: 452.

2 Garnett RL, MacIntyre A, Lindsay P, et al. Perioperative ischaemia in aortic surgery: combined epidural/general anaesthesia and epidural analgesia vs general anaesthesia and $i v$ analgesia. Can J Anaesth 1996; 43: 769-77.

3 Garnett $R L$. Combined epidural/general anaesthesia (Letter-reply). Can J Anaesth 1997; 44: 452-3.

4 Bromage PR. 50 years on the wrong side of the reflex arc (Editorial). Reg Anesth 1996; 21(6S): 1-4.

5 Bromage PR, Camporesi E, Chestnut D. Epidural narcotics for postoperative analgesia. Anesth Analg 1980; 59: $473-80$.

\section{REPLY}

Thank you for the opportunity to respond to the comments/criticisms raised by Dr. Bromage. My reply, not retort, to Dr. Carli that "nothing was further from the truth, ${ }^{2}$ is valid. ${ }^{2}$ The most stimulating event in surgery is the incision. In our experience, all patients undergoing aortic surgery under general anaesthesia respond to the incision and require more opioids or deeper inbalational anaesthesia. ${ }^{3}$ None of our epidural patients required additional opioids at the time of incision. To me, this is the acid test and demonstrated that the epidural was working and the block extended to the xyphoid process. Pancuronium may mask motor responses to inadequate segmental analgesia, but all anaesthetic drugs mask response to pain in some way.

We did not verify the height of the block before or after surgery. We relied on patient comfort as the measure of success as I am sure Dr. Bromage does in his clinical prac- tice. Demonstration of a segmental level of blockade does not guarantee success as frequently demonstrated at caesarean section. Postoperatively, it may be impossible to demonstrate segmental block using bupivacaine $0.1 \%$ in our geriatric patient population. In fact, we reduced the concentration of bupivacaine from $0.125 \%$ to $0.1 \%$ because of sometimes persistent motor block. Analgesia continued, the patients were comfortable, and this was the aim.

If I were to repeat this study, the one change would be to demonstrate a T5 block before induction of anaesthesia. I am sure we achieved this level, but not to perify it leads to unanswerable questions.

\section{R.L. Garnett MD FRCPC \\ Ottawa, Ontario}

\section{REFERENCES}

1 Carli F, Klubien K, Baker C. Combined epidural/general anaesthesia (Letter). Can J Anaesth 1997; 44: 452

2 Garnett $R L$. Combined epidural/general anaesthesia (Letter-reply). Can J Anaesth 1997; 44: 452-3.

3 Garnett $R L$, MacIntyre $A$, Lindsay $P$, et al. Perioperative ischaemia in aortic surgery: combined epidural/general anaesthesia and epidural analgesia vs general anaesthesia and $i v$ analgesia. Can $J$ Anaesth $1996 ; 43: 769-77$

\section{Combined spinal/epidural in neonates and children}

To the Editor:

I have two concerns regarding the technique of Drs Williams et al. regarding the use of combined spinal and epidural anesthesia in the awake neonate and infant. ${ }^{1}$ First is the recommendation of performing an epidural block after a spinal anaesthetic. The authors failed to mention the possibility of total spinal block from the passage of local anaesthetic through the hole in the dura. ${ }^{2}$

Second, I wonder about the advisability of performing extensive surgical procedures without airway protection although the authors previously described the use of spinal anesthesia as the sole anaesthetic for repair of gastroscises ${ }^{3}$ and closure of meningomyelocele. ${ }^{4}$ The maintenance of spontaneous ventilation may be further compromised by the surgical procedure as well as the need for supplemental intravenous sedation. The authors state that extubation may not be possible with the combined technique. We performed continuous caudal epidural anaesthesia with chloroprocaine combined with general anaesthesia in 25 neonates and 